\title{
Position-Selective Growth of ZnO Nanowires by Ultrasonic Spray Pyrolysis
}

\author{
Myo Than Htay ${ }^{\mathrm{a}}$, Yoshio Hashimoto ${ }^{\mathrm{a}}$, Noritaka Momose $^{\mathrm{b}}$, Kentaro Ito $^{\mathrm{a}}$ \\ ${ }^{a}$ Department of Electrical and Electronic Engineering, Faculty of Engineering, Shinshu University, 4-17-1 Wakasato, \\ Nagano 380-8553, Japan \\ ${ }^{b}$ Department of Electrical and Electronic Engineering, Nagano National College of Technology, 716 Tokuma, Nagano \\ 381-8550, Japan
}

\begin{abstract}
Position-selective growth of $\mathrm{ZnO}$ nanowires was realized by utilizing a patterned soda-lime glass layer. The soda-lime glass layer contributes as the origin of nucleation sites for the growth of nanowires in economically viable ultrasonic spray pyrolysis technique. The formation of nanowires took place with good reproducibility at relatively low substrate temperatures of 250$400^{\circ} \mathrm{C}$ when the soda-lime glass matrix was present at the surface of substrates, and such a nitrate compound as nitric acid or nitrate salt was mixed in the precursor solutions. Based on this technique, the position-selective growth and density-controlled growth of $\mathrm{ZnO}$ nanowires can be performed on various types of substrate. The presence of a $\mathrm{CaO}$ compound in the glass matrix was revealed as an important condition to grow the nanowires.
\end{abstract}

Keywords: ZnO, Nanowire, Ultrasonic spray pyrolysis, Position-selectivity, Growth mechanism

PACS: 81.05.Dz, 81.07.-b, 81.16.-c, 81.20.-n, 81.20.Rg

\section{Introduction}

$\mathrm{ZnO}$, a wide band gap $(3.37 \mathrm{eV}$ at $300 \mathrm{~K})$ semiconductor [1], is known to have various nanostructures such as nanowires (NWs), nanorods, nanobelts, nanoplatelets, and so on. Due to their particular characteristics, these nanostructures have advantages for use in the future nanoelectronic, nanophotonic, and photovoltaic devices. Recently, there has been a lot of interest in functional devices based on $\mathrm{ZnO} \mathrm{NWs}$ like a NW transistor [2], NW laser [3], field emission devices [4], dye-sensitized photovoltaic cells [5], NWs generator [6], and so on. However, the lack of controllability in growth process of $\mathrm{ZnO}$ NWs has held back the use of this material in developing these devices for practical applications. The techniques to control the growth parameters of $\mathrm{ZnO}$ NWs such as growth position, growth orientation, and aspect ratio of NWs remain a significant challenge. Up to now, there are only a few reports that discuss controlling the growth position of $\mathrm{ZnO}$ NWs. Huang et al. reported on a well controlled position-selective growth of $\mathrm{ZnO} N W$ s array from a gold-patterned substrate using vapor transport technique [7]. This growth process was based on the vapor-liquid-solid (VLS) growth mechanism, which is well known for the formation of one-dimensional crystal such as whiskers $[8,9]$. In this technique, due to the usage of Au metal catalyst, relatively high growth temperature of about $800^{\circ} \mathrm{C}$ is necessary to obtain the NWs, and the contamination problem caused by the metal catalyst used is inevitable. 
Other interesting approaches like hydrothermal technique provides the growth of NWs at a temperature as low as $60^{\circ} \mathrm{C}$. However, in this technique, the crystal growth rate is very slow so that it needs a lengthy growth period (several hours), and post-annealing process at a much higher temperature is also necessary to improve the crystal quality $[6,10]$. Besides, controlling the growth position of NWs by this technique is still a subject to overcome.

In this paper, we report on an economically viable technique to realize the position-selective growth of $\mathrm{ZnO} \mathrm{NWs}$ on various substrates utilizing ultrasonic spray pyrolysis (USP) technique. This technique ensures the position-selective growth of $\mathrm{ZnO}$ NWs at a temperature of $250-400^{\circ} \mathrm{C}$ without using an expensive metal catalyst such as Au. The growth temperature is low enough to be compatible with the fabrication processes of semiconductor devices. As an inexpensive and time-saving deposition method, it has the promising advantage of being applied to the largearea fabrication of electronic display panels and thin film solar cells. We also proposed a new mechanism to explain the position-selective growth of $\mathrm{ZnO} \mathrm{NWs}$, based on the finding that the growth is closely associated with the usage of soda-lime glass matrix (SGM) and nitrate ions in the USP technique.

\section{Experimental details}

The details of our original USP system for growing $\mathrm{ZnO}$ NWs were described elsewhere [11]. In our technique, zinc acetate dihydrate (Wako, 99\%) was used as a zinc source. Deionized water was used as a solvent of the precursor solution, and also as the source of oxygen. The $\mathrm{pH}$ of the precursor solution was adjusted by mixing ammonium acetate (Wako, 97\%) [12]. Incorporation of ammonium acetate in the solution also enhances the crystallization of $\mathrm{ZnO}$ so that the growth of good quality NWs at relatively low temperature is obtained in this USP technique [13]. Growth of ZnO NWs was realized by using an SGM as a nucleation layer. A soda-lime glass (SLG, MATSUNAMI GLASS, S-7213) was used as the source of SGM. The chemical composition of SLG is shown in table 1 . The content of $\mathrm{CaO}$ and $\mathrm{Na}_{2} \mathrm{O}$ in SLG is 9.6 and 13.1 in weight percent (Wt.\%), respectively. These alkaline-earth oxides $(\mathrm{CaO})$ contribute as the nucleation sites for the growth of NWs, and are activated by precursor mist, which contains a suitable amount of nitric acid or nitrate salt. Conventional photolithography process was applied to the substrate for patterning the SGM in order to control the growth position of NWs. For preparing a high definition pattern, focused ion beam microfabrication (FIB, Seiko Instruments Inc., SMI-2050, $\mathrm{Ga}^{+}$ion) process was used as well.

The following analyses were conducted for evaluating various properties of material. A field emission scanning electron microscope (FE-SEM, Hitachi, S-4100) was used to investigate the surface morphology of the samples. A transmission electron microscopy (TEM, JEOL, JEM$2010, \mathrm{LaB}_{6}$ cathode) and selected area electron diffraction (SAED) analysis were carried out at an acceleration voltage of $200 \mathrm{kV}$ to study the details of crystalline nature of NWs. The samples for TEM analysis and cross-sectional observation were prepared by using FIB microfabrication process.

\section{Results and Discussion}

To study the morphological transformation of ZnO NWs during the crystal growth process, a microscopic observation was conducted on the samples prepared during different growth periods of 15,30 and $60 \mathrm{~min}$ at the growth temperature of $400^{\circ} \mathrm{C}$. The corresponding SEM images 
are shown in Fig. 1. In Fig. 1a, which represents an early crystal growth phase, only a thin layer composed of small crystal grains is observed. We may call this layer a self-assembled seeding layer (SL). As the growth period become longer, crystal islands which we may call seed crystallites (SCs) are formed on the SL with various orientations as shown in Fig. 1b. After that, one-dimensional growth takes place on these SCs as can be seen in Fig. 1c, eventually resulting in the growth of NWs. By careful observation, it is noted that the crystallographical growth direction of NWs depends mainly on the crystal orientation of SCs, i.e., the shape of wire tip is defined by that of earlier protruded SCs. The preferred growth direction of NWs are found to be in the [0001], [1010], and [1120] crystallographic directions of wurtzite crystal. The details of crystalline and optical properties of NWs were reported in our previous works $[11,12,13]$. The similar morphological transformation was also observed in the growth temperatures of $250^{\circ} \mathrm{C}$ and $300^{\circ} \mathrm{C}$, respectively. But the growth size of individual SC was much smaller at the lower temperatures so that relatively thinner NWs were obtained [11].

To investigate the growth mechanism, a group of experiments were conducted. The growth temperature was fixed at $300^{\circ} \mathrm{C}$, a growth period at $60 \mathrm{~min}$, and a flow rate of conveying gas $\left(\mathrm{N}_{2}\right)$ at $1.4 \mathrm{~L} / \mathrm{min}$. We first concentrated our attention to the growth behavior of NWs crystal on different substrates. The effect of ingredients in precursor solutions upon the formation of onedimensional crystal was then studied. The details of precursor solutions used in the experiments are shown in table 2. Table 3 shows the results of these experiments. In the table, the letters "n", "P", and "S" stand for the results that yield "no growth of one-dimensional NWs", "poor growth of NWs with low reproducibility", and "stable growth of NWs with high reproducibility", respectively. The letter " $o$ " represents the omitted experiment, of which no prominent result is expected.

From the results, it is clearly noticed that the formation of one-dimensional crystal, i.e., the growth of NWs takes place with good reproducibility only when SGM is present at the surface of substrates, and a nitrate compound such as nitric acid or nitrate salt is mixed in the precursor solutions. On the other hand, there is no formation of NWs on any kinds of substrate when using solution A or B in which the nitrate compound is not contained. This shows the importance of SGM and nitrate compound for the growth of NWs. In other words, the alkaline-earth species such as calcium oxide originated from SGM is necessary near the substrate surface and may be related with the formation of SCs on which the growth of NWs takes place. Along with this, the introducing of nitrate species from the precursor mist also plays an important role since the growth of NWs is not obtained without it. In comparing the results using solutions $\mathrm{C}$ and $\mathrm{D}$, there are no obvious differences between them. This indicates that indium is not the key reagent for the growth of NWs. In the case of solution E and G in which calcium nitrate is contained, formation of NWs is realized on all kinds of substrates. However, the reproducibility is poor when using substrates without SGM. From these results, we consider that the formation of SCs is catalyzed more effectively when calcium-related centers are embedded at the surface of SGM, rather than being delivered from the salt dissolved in the precursor solution. When solution $\mathrm{F}$ containing sodium nitrate is used, the formation of NWs is not efficient compared to the solution containing calcium nitrate, showing that calcium nitrate is the most important compound for the growth of NWs on any substrates used.

Since, the use of a metal catalyst is not a compulsory condition for synthesizing one-dimensional wires, there may be differences in our technique to those based on the well-known VLS mechanism, in which a metal catalyst is a necessary condition to establish the so-called vapor-liquidsolid interfaces during the growth process. In the following we propose an alternate growth model that is consistent with our observations. The nitrate compound contained in the mist of 
a precursor solution will thermally decomposed into nitrogen oxide molecules as the mist approaches the surface of a heated substrate. When the substrate consists of calcium oxide, there are basic $\mathrm{O}^{2-}$ sites on the surface due to crystal defects. $\mathrm{NO}_{2}^{2-}$ radical species are thus formed at the sites as elucidated by electron paramagnetic resonance $[14,15]$. This reaction is considered as the first step to nucleate the NW growth, possibly giving rise to a decrease in the potential energy of the site so that it could behave as an effective absorption center for the reactants such as zinc and oxygen. The radical species then accommodate the zinc substituting for the nitrogen oxide. Oxidation of the zinc species eventually forms a seed crystal: it grows in a preferred crystallographic direction, protruding from the site with a much faster rate than at an area without the sites. One-dimensional crystalline NWs will be thus obtained. At the initial stage of the growth, in particular, the reactant species might be physically adsorbed on the SL and then transported to the nearby nucleation sites by surface diffusion.

In Fig. 2, bright field TEM images and corresponding SAED patterns of a cross-sectional sample are shown. In this sample, part of the SLG substrate was coated with about a $100 \mathrm{~nm}$ thick Al metal layer to passivate the reaction site on $\mathrm{CaO}$. Preparation of NWs was carried out by using solution $\mathrm{C}$ at $300^{\circ} \mathrm{C}$ and the growth period of $60 \mathrm{~min}$. Fig. $2 \mathrm{~b}$ is an enlarged image of the squared region shown in Fig. 2a. It can be clearly seen that the formation of NWs takes place exclusively on the SGM region which is exposed to the vapor source, while on the metal film there is only a thin $\mathrm{ZnO}$ layer, instead. This layer is about two times thicker than the SL formed on the SGM. By careful observation, it is revealed that the total volume of NWs together with the SL is approximately equal to that of the layer deposited on the metal film. This implies that a part of the vapor sources which impinge upon and are then chemically absorbed on the SGM is consumed for the NW growth. In order to confirm this result, we repeated similar experiments several times by using the SLG substrate that is capped partly with a metal film such as $\mathrm{Al}$ and Mo in different patterns. However, almost the same results as shown in Fig. 2 were obtained in all the experiments. This is an important clue to understand how NWs are grown on the SGM selectively. As we suggested above, the growth of NWs can be obtained only when CaO-related nucleation sites are created on the substrate surface. A part of the reactants absorbed on the SGM are consumed in the formation of SL, while the rest of it is contributed to the growth of SCs and NWs. This type of growth mechanism is not prevalent on the metal surface because there are no nucleation sites: only a thicker polycrystalline $\mathrm{ZnO}$ film is deposited here.

Figure $2 \mathrm{c}$ shows the base region of a nanowire together with the SL. It clearly shows that the nanowire is grown on a thin layer (about $30 \mathrm{~nm}$ thick) which is formed in the initial phase of growth process. The corresponding SAED patterns of encircled regions (1), (2) and (3) are described at the bottom of Fig. 2c. It is to be noted that a polycrystalline phase observed at the very base region of the nanowire transforms to a monocrystalline one when we look at the diffraction patterns obtained from regions (3) to (1). All of the diffractions are identified with a wurtzite structure. Region (3), which represents the SL, is composed of small ZnO crystal grains as expected from the TEM image. Since a diffraction pattern with sixfold symmetry is obtained from region (1), the monocrystalline nanowire observed in the image is grown along the [10 $\overline{1} 0]$ crystallographic direction of wurtzite crystal. The transformation of crystalline nature at the base of NWs is quite interesting because it could be traced back to the initial formation of SCs.

Based on the mechanism discussed above, we demonstrate the position-selective growth of NWs by controlling the position and/or geometry of an SGM region on the substrate. The fabrication procedure for a sample is shown in Fig. 3a. Firstly, the substrate containing SGM is coated with a metallic passivation layer. Then, the layer is fabricated to a desired pattern using FIB or photolithography. This pattern defines an exposed SGM region on the metallized substrate 
surface. Finally, the position-selective growth of NWs is completed using our USP technique. The growth conditions are as follows: the growth temperature is $300^{\circ} \mathrm{C}$, the growth period is 60 $\mathrm{min}$, and the solution used belongs to category C. In Fig. $3 \mathrm{~b}$ and $3 \mathrm{c}$, the images before and after growth of NWs are shown, respectively. Here, the pattern (grooves in the image) in Fig. $3 \mathrm{~b}$ is formed by etching off the metal layer utilizing FIB. From the result as shown in Fig. 3c, it is clearly found that the NWs grow densely along the SGM grooves but not on the metal layer. However, it is appears that some wires are growing outside the boundary of the pattern due to the orientation of bending wires that has tendency of spreading outwards from their growth base. The position-selectivity is obtained with high reproducibility in various patterns fabricated by photolithography process using difference types of passivation layer such as $\mathrm{Al}$ (about $100 \mathrm{~nm}$ and $400 \mathrm{~nm}$ thick) and Mo (about $150 \mathrm{~nm}$ thick) as shown in Fig. 3d, 3e and 3f, respectively. These results firmly support our proposal discussed above. In principle, the position-selective growth of NWs can be applied to any patterns and substrates having SGM at the surface by using this technique. The accuracy of position-selectivity is of the order of micrometer at present. It could be made possible in the future to control the growth position of even a single nanowire by improving microfabricaion techniques of SGM. The formation of bending wires is still a problem to overcome in order to realize a high accuracy positional control.

We conducted another experiment to examine the controllability of the growth density of NWs by applying the growth mechanism proposed above. The concentration of SGM exposed at the surface of substrate is indirectly controlled by adjusting the amount of SLG deposited onto the substrate utilizing RF magnetron sputtering. Here, the amount of deposited SLG is controlled in term of thickness by the sputter rate. In Fig. 4d, the procedure for preparing the samples is shown. Corning 7059 glass, which contained less than $0.1 \mathrm{Wt} \%$ of alkaline species was used as the substrate for this experiment so that unintended incorporation of alkaline species is negligible. After coating a metal layer such as Mo on the corning 7059 substrate, SGM is deposited with a controlled thickness. Growth of NWs is performed at $300^{\circ} \mathrm{C}$ for $60 \mathrm{~min}$ using solution $\mathrm{C}$ in this experiment. Fig. $4 \mathrm{~b}$ and $4 \mathrm{c}$ are the images of NWs grown on substrates having SGM equivalent to $5 \mathrm{~nm}$ and $2 \mathrm{~nm}$ in thickness, respectively. It is found that the growth density of NWs is decreased as the amount of deposited SGM is reduced. The average growth density is about 20 and 10 wires per $100 \mu \mathrm{m}^{2}$. In comparison, the image of NWs grown directly on the SLG substrate which contained $9.6 \mathrm{Wt} . \%$ of $\mathrm{CaO}$ is shown in Fig. $4 \mathrm{a}$. Since the surface concentration of $\mathrm{CaO}$ in this sample is much higher, the average growth density is about 600 wires per 100 $\mu \mathrm{m}^{2}$. The result shows that the control of growth density of NWs can be realized by adjusting the concentration of SGM exposed at the surface of substrate. A magnified cross-sectional image of the sample shown in Fig. $4 \mathrm{~b}$ is described in Fig. 4e. In the image, there are bundles of NWs that confined locally on the SL. It is noticed that the appearance of a NWs bundle is similar to that of the grass which grows from its own root. This growth nature of NWs is different from the one grown directly on SLG substrate. This may be due to the difference in the confinement of alkaline-earth species on the Mo metal surface with that of the SGM. In this sample, the $5 \mathrm{~nm}$ thick SGM is considered to be deposited in the form of islands rather than a continuous layer on the Mo metal layer. The grass-like bundles of NWs are formed presumably because their growth is limited to the sites where SGM islands are located.

\section{Conclusions}

An economically viable technique to realize the position-selective growth of $\mathrm{ZnO}$ nanowires was demonstrated by utilizing a home-made ultrasonic spray pyrolysis system. In this technique, 


\begin{tabular}{|c|c|}
\hline Compound & Wt.\% \\
\hline $\mathrm{SiO}_{2}$ & 71.7 \\
\hline $\mathrm{Al}_{2} \mathrm{O}_{3}$ & 0.2 \\
\hline $\mathrm{CaO}$ & 9.6 \\
\hline $\mathrm{Na}_{2} \mathrm{O}$ & 13.1 \\
\hline $\mathrm{K}_{2} \mathrm{O}$ & - \\
\hline $\mathrm{BaO}$ & - \\
\hline $\mathrm{Fe}_{2} \mathrm{O}_{3}$ & 0.1 \\
\hline $\mathrm{MgO}$ & 4.4 \\
\hline $\mathrm{SO}_{3}$ & 0.4 \\
\hline $\mathrm{B}_{2} \mathrm{O}_{3}$ & - \\
\hline $\mathrm{ZnO}$ & - \\
\hline
\end{tabular}

the position-selectivity is realized at relatively low temperatures of $250-400^{\circ} \mathrm{C}$ by using a thin film soda-lime glass matrix as a nucleation layer. The growth dynamics of nanowires and the mechanism of the position-selectivity were discussed in details based on the experimental results. The transformation of crystalline nature at the base of $\mathrm{ZnO}$ nanowires was observed by selected area electron diffraction analysis. The growth of nanowires was obtained after the formation of seed crystallites possibly via the surface diffusion of adsorbed reactants on the self-assembled seeding layer. When soda-lime glass was used as a substrate, and while nitrate species such as a nitric acid or a nitrate salt is mixed in the precursor solution, the position-selective growth of nanowires was realized with high reproducibility. The basic sites of the $\mathrm{CaO}$ surface might play a crucial role to nucleate $\mathrm{ZnO}$ seed crystals. Controlling the growth density of nanowires was demonstrated by adjusting the concentration of $\mathrm{CaO}$ on the growth surface. To summarize as a whole, the position-selective growth and density-controlled growth of $\mathrm{ZnO}$ nanowires could be performed on various types of substrate at a relatively low temperature that is compatible with other device fabrication processes by applying ultrasonic spray pyrolysis technique.

\section{Acknowledgements}

One of the authors (M. T. Htay) would like to express a deep gratitude to the Ministry of Education, Culture, Sports, Science and Technology of Japan for grant of postgraduate scholarship. Special thanks are due to Mr. Isamu Minemura for many help with construction of experimental apparatus and Mr. Tomohiko Yamakami for TEM photography.

\section{References}

[1] N. Izyumskaya, V. Avrutin, Ü. Özgür, Y. I. Alivov, and H. Morkoç, Phys. Stat. Sol. B, 244 (2007) 1439.

[2] H. D. Xiong, W. Wang, Q. Li, C. A. Richter, J. S. Suehle, W. K. Hong, T. Lee, D. M. Fleetwood, Appl. Phys. Lett., 91 (2007) 053107.

[3] C. J. Lee, T. J. Lee, S. C. Lyu, Y. Zhang, H. Ruh, and H. J. Lee, Appl. Phys. Lett. , 81 (2002) 3648.

[4] M. H. Haung, S. Mao, H. Feick, H. Yan, Y. Wu, H. Kind, E. Weber, R. Russo, and P. Yang, Science, 292 (2001) 1897 .

[5] M. Law, L. E. Greene, J. C. Johnson, R. Saykally, and P. Yang, Nature Mater. , 4 (2005) 455.

[6] Y. Qin, X. Wang, and Z. L. Wang, Nature, 451 (2008) 809.

[7] M. H. Huang, Y. Wu, H. Feick, N. Tran, E. Weber, and P. Yang, Adv. Mater. , 13 (2001) 113. 


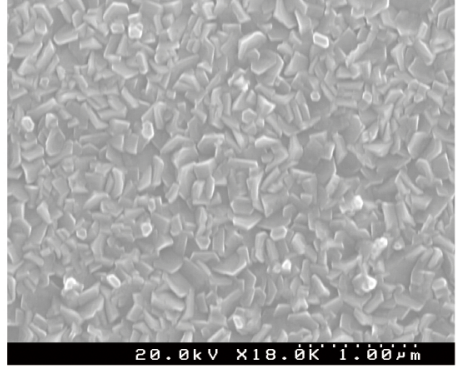

(a)

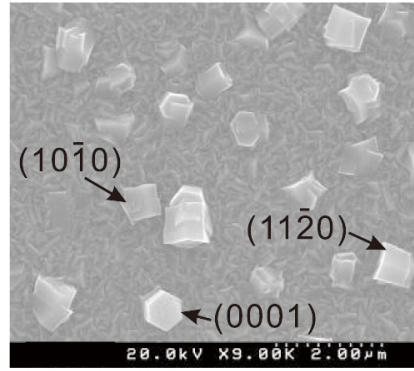

(b)

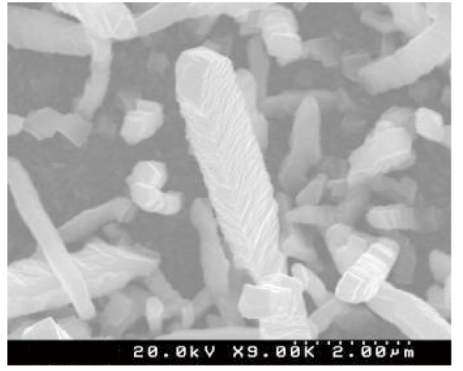

(c)

Figure 1: FE-SEM images showing the shape transformation of $\mathrm{ZnO} N W$ s prepared at $400^{\circ} \mathrm{C}$ for growth period of (a) 15 min, (b) $30 \mathrm{~min}$, and (c) $60 \mathrm{~min}$, respectively.

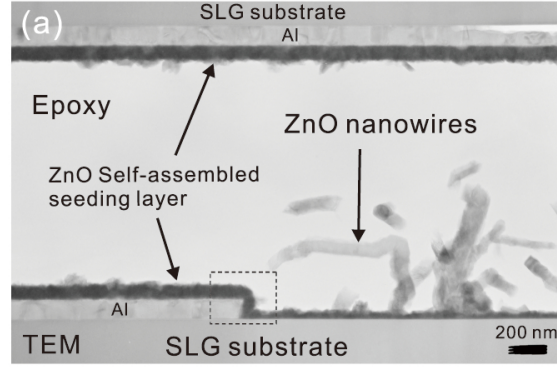

(b)

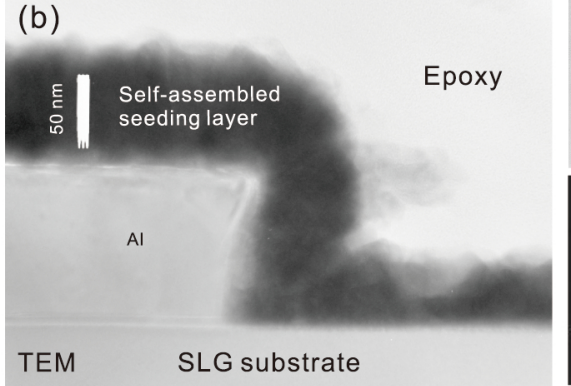

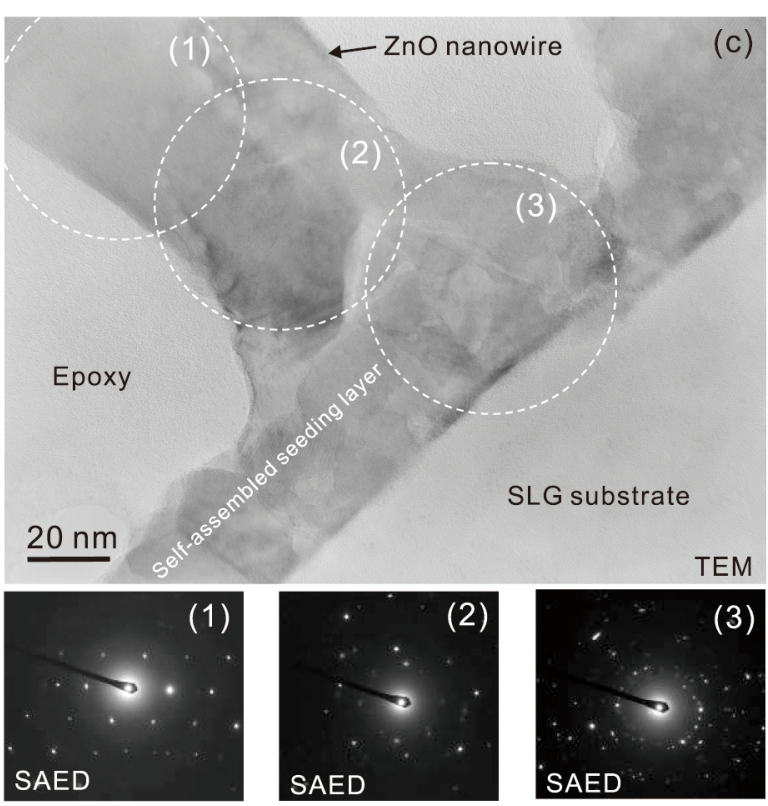

(c)

EM

Figure 2: (a) Cross-sectional bright field TEM image of SL and NWs. (b) Enlarged image of squared region shown in (a). (c) Cross-sectional TEM image of the base of nanowire and SL. Corresponding SAED patterns of regions (1), (2), and (3) are shown at the bottom. 


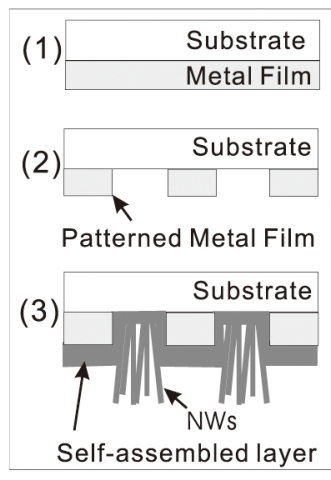

(a)

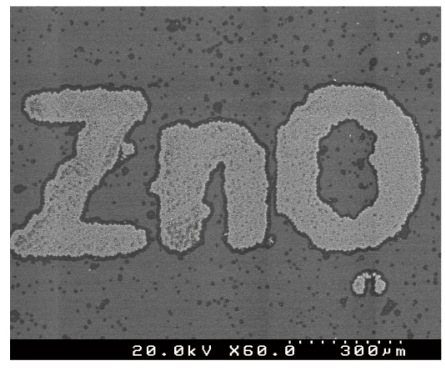

(d)

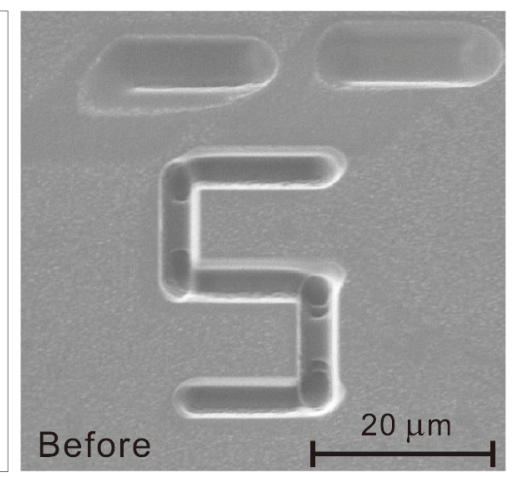

(b)

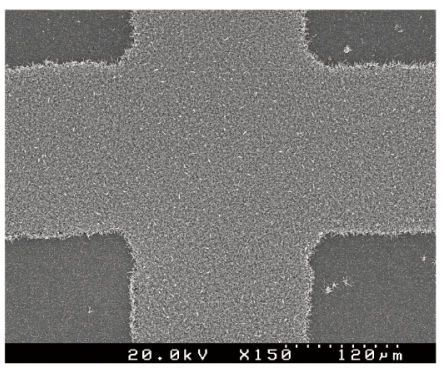

(e)

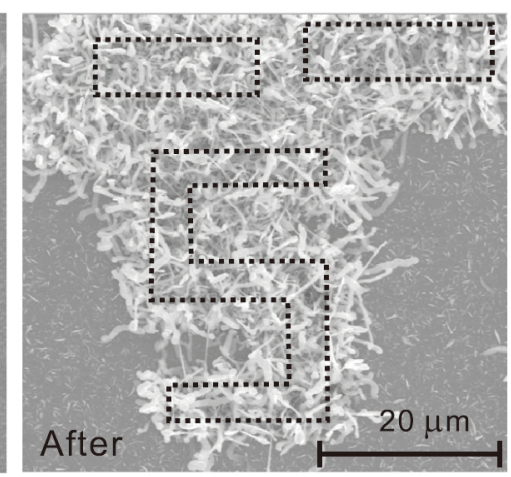

(c)

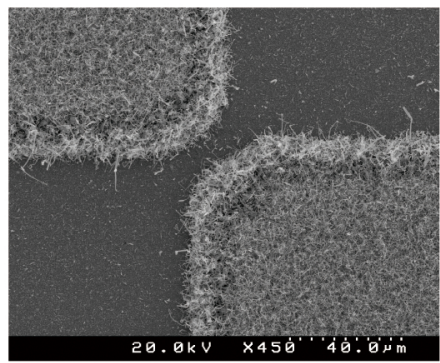

(f)

Figure 3: (a) Steps for preparing position-selective growth of NWs. (b) A pattern etched off by FIB. (c) Position-selective growth of NWs on the pattern shown in (b). Position-selective growth of NWs on the substrates with passivation layer of (d) $100 \mathrm{~nm}$ thick Al, (e) $400 \mathrm{~nm}$ thick Al, and (f) $150 \mathrm{~nm}$ thick Mo patterned by photolithography process. 


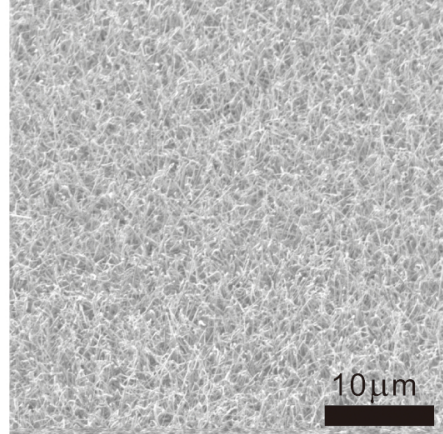

(a)

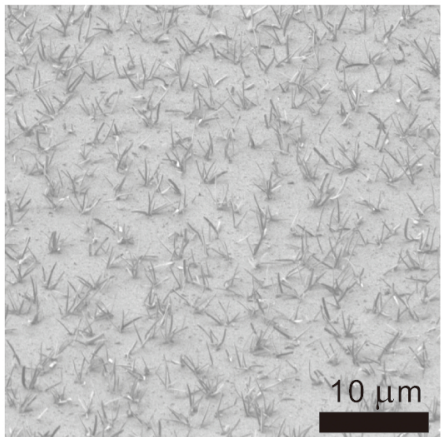

(b)

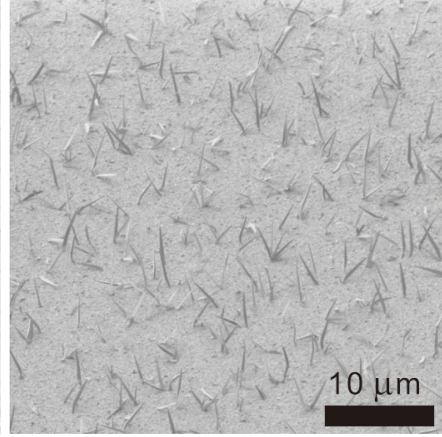

(c)

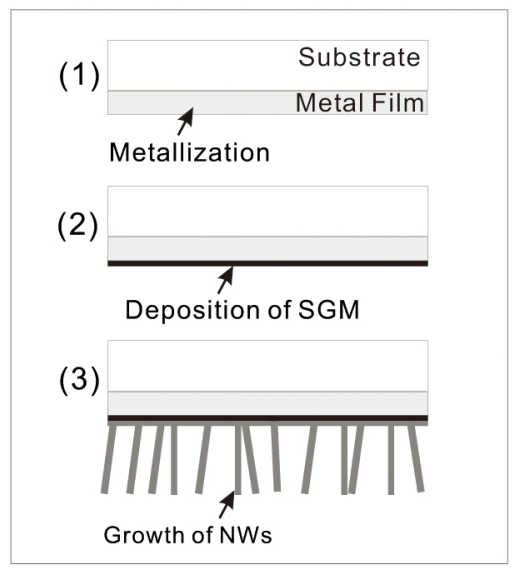

(d)

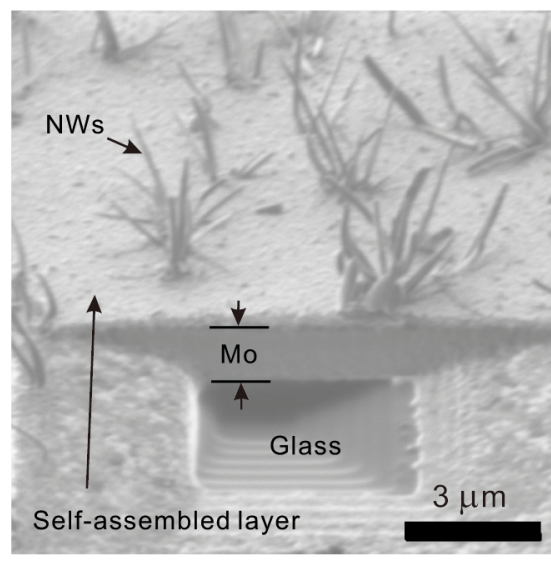

(e)

Figure 4: Images of NWs grown on (a) SLG subtrate, (b) Mo/corning 7059 substrate coated with SGM $5 \mathrm{~nm}$, and (c) 2 $\mathrm{nm}$ in thickness. (d) Procedure for preparing the density-controlled growth of NWs. (e) Magnified cross-sectional image of the sample shown in (b). 
Table 2: The details of precursor solutions.

\begin{tabular}{ccl}
\hline \hline Types of precursor solution & Solvent & Solutes (mole ratio) \\
\hline $\mathrm{A}$ & $\mathrm{H}_{2} \mathrm{O}$ & $\mathrm{ZA}(0.3 \mathrm{~mol} / \mathrm{L})$ \\
$\mathrm{B}$ & $\mathrm{H}_{2} \mathrm{O}$ & $\mathrm{ZA}+\mathrm{AA}(1: 5)$ \\
$\mathrm{C}$ & $\mathrm{H}_{2} \mathrm{O}$ & $\mathrm{ZA}+\mathrm{AA}+\mathrm{NA}(10: 50: 1)$ \\
$\mathrm{D}$ & $\mathrm{H}_{2} \mathrm{O}$ & $\mathrm{ZA}+\mathrm{AA}+\mathrm{IN}(10: 50: 1)$ \\
$\mathrm{E}$ & $\mathrm{H}_{2} \mathrm{O}$ & $\mathrm{ZA}+\mathrm{AA}+\mathrm{CN}(1: 5: 2)$ \\
$\mathrm{F}$ & $\mathrm{H}_{2} \mathrm{O}$ & $\mathrm{ZA}+\mathrm{AA}+\mathrm{SN}(1: 5: 2)$ \\
$\mathrm{G}$ & $\mathrm{H}_{2} \mathrm{O}$ & ZA+AA+CN+SN $(1: 5: 2: 2)$ \\
\hline ZA: Zinc Acetate; & AA: Amonium Acetate; & NA: Nitric Acid \\
IN: Indium Nitrate; & CN: Calcium Nitrate; & SN: Sodium Nitrate
\end{tabular}

Table 3: The effect of substrates and precursor solutions on the growth behavior of ZnO NWs.

\begin{tabular}{lccccccc}
\hline Substrate & \multicolumn{1}{c}{ Types of precursor solution } \\
& $\mathrm{A}$ & $\mathrm{B}$ & $\mathrm{C}$ & $\mathrm{D}$ & $\mathrm{E}$ & $\mathrm{F}$ & $\mathrm{G}$ \\
\hline $\mathrm{SLG}$ & $n$ & $n$ & $\mathbf{S}$ & $\mathbf{S}$ & $\mathbf{S}$ & $\mathrm{P}$ & $\mathbf{S}$ \\
7059 & $n$ & $n$ & $n$ & $n$ & $\mathrm{P}$ & $n$ & $\mathrm{P}$ \\
1737 & $n$ & $n$ & $n$ & $n$ & $\mathrm{P}$ & $o$ & $o$ \\
$\mathrm{Si}(001)$ & $n$ & $n$ & $n$ & $n$ & $\mathrm{P}$ & $o$ & $o$ \\
$\mathrm{SiO} / \mathrm{Si}$ & $n$ & $n$ & $n$ & $n$ & $\mathrm{P}$ & $o$ & $o$ \\
$\mathrm{ZnO} / 7059$ & $n$ & $n$ & $n$ & $n$ & $\mathrm{P}$ & $o$ & $o$ \\
$\mathrm{Mo} / 7059$ & $n$ & $n$ & $n$ & $n$ & $\mathrm{P}$ & $o$ & $o$ \\
$\mathrm{Al} / 7059$ & $o$ & $o$ & $n$ & $n$ & $\mathrm{P}$ & $o$ & $o$ \\
$\mathrm{SLG} / \mathrm{Mo} / 7059$ & $o$ & $o$ & $\mathbf{S}$ & $\mathbf{S}$ & $o$ & $\mathrm{P}$ & $\mathbf{S}$ \\
$\mathrm{SLG} / \mathrm{Al} / 7059$ & $o$ & $o$ & $\mathbf{S}$ & $\mathbf{S}$ & $\mathbf{S}$ & $o$ & $o$ \\
\hline
\end{tabular}

[8] R. S. Wagner and W. C. Ellis, Appl. Phys. Lett. , 4 (1964) 89.

[9] K. Hiruma, M. Yazawa, T. Katsuyama, K. Ogawa, K. Haraguchi, M. Koguchi, and H. kakibayashi, J. Appl. Phys. , 77 (1995) 447.

[10] Z. R. Tian, J. A. Voigt, J. Liu, B. Mckenzie, M. J. Mcdermott, M. A. Rodriguez, H. Konishi, and H. Xu, Nature Mater. , 2 (2003) 821

[11] M. T. Htay, Y. Hashimoto, and K. Ito, Jpn. J. Appl. Phys., 46 (2007) 440.

[12] M. T. Htay, Y. Tani, Y. Hashimoto, and K. Ito, J. Mater. Sci: Mater. Electron., 20 (2009) 341.

[13] M. T. Htay, M. Itoh, Y. Hashimoto, and K. Ito, Jpn. J. Appl. Phys., 47 (2008) 541.

[14] M. C. Paganini, M. Chiesa, F. Dolci, P. Martino, and E. Giamello, J. Phys. Chem.B, 110 (2006) 11918.

[15] M. C. Paganimi, M. Chiesa, P. Martino, and E. Giamello, J. Phys. Chem.B, 106 (2002) 12531. 